\title{
ARTICLE \\ Effect of exogenous testosterone on cooperation depends on personality and time pressure
}

\author{
Brian M. Bird ${ }^{1}$, Shawn N. Geniole ${ }^{2,3}$, Tanya L. Procyshyn ${ }^{4}$, Triana L. Ortiz ${ }^{2}$, Justin M. Carré ${ }^{2}$ and Neil V. Watson ${ }^{1}$
}

The social heuristic hypothesis posits that human cooperation is an intuitive response that is expressed especially under conditions of time-constraint. Conversely, it proposes that for individuals given an opportunity for reflection, cooperation is more likely to be curtailed by an optimizing process calibrated to maximize individual benefit in a given situation. Notably, the steroid hormone testosterone has also been implicated in intuitive decision-making, including both prosocial and anti-social behaviors, with effects strongest in men with particular dispositional characteristics. This raises the possibility that increased testosterone may augment the effects predicted by the social heuristic hypothesis, particularly among men higher in specific dispositional characteristics (dominance, impulsivity, independent self-construal: high risk for testosterone-induced antisocial behavior). Here, in a testosterone administration study with a relatively large sample of men $(N=400)$, we test this possibility in a double-blind, placebo-controlled paradigm, with men randomly assigned to play a one-shot public goods game either under time-pressure (forced intuition) or with a time delay (forced reflection). Results revealed that within the placebo group, time-pressure (versus forced delay) increased cooperation among low risk men, but decreased cooperation among high risk men. Testosterone further moderated this pattern by abolishing the time-pressure effect in low risk men and-in high risk men-reversing the effect by selectively reducing offers (compared to placebo) under forced delay. This is the first evidence that testosterone and personality can interact with timepressure and delay to predict human cooperation.

Neuropsychopharmacology (2019) 44:538-545; https://doi.org/10.1038/s41386-018-0220-8

\begin{abstract}
"The most powerful force ever known on this planet is human cooperation - a force for construction and destruction" Jonathan Haidt
\end{abstract}

Cooperation is a defining feature of human social interaction [1], yet it remains a perplexing phenomenon: to cooperate with others confers benefits to the group as a whole, but because such an act necessarily involves a personal cost, it also creates the temptation to withhold cooperation in the hopes of benefitting from others' prosociality [2]. Humans further possess the capability to switch from selfless to self-interested behaviors (and vice-versa) in relatively short periods of time, raising the question as to what mechanisms act upon this behavior. Such dynamics have inspired considerable research efforts to delineate the ultimate and proximate factors that influence human decisionmaking on whether or not to cooperate.

\section{SOCIAL HEURISTIC HYPOTHESIS AND DECISION-MAKING FRAMEWORKS}

The social heuristic hypothesis posits that human intuitions determine if our default response is selfish or cooperative, with such intuitions shaped by daily experiences [1, 3]. Cooperation, it is argued, is advantageous in daily life: in our repeated interactions with others, reputational status is at stake, accompanied by the possibility of sanctions for good or bad behavior, and thus we can successfully navigate these social interactions by cooperating. As a function of these daily experiences, humans can develop cooperative intuitions $[3,4]$. Of course, some individuals cooperate for other reasons, such as to increase their own self-regard (typically referred to as "warm glow" $[5,6]$ ), but we review the literature on the social heuristic hypothesis, which describes the functional utility of cooperating in daily life, as this literature specifically helped inform our hypotheses in the present study. The social heuristic hypothesis builds on a larger dual-process framework, arguing that our decisions arise as a function of either (a) automatic, fast, rigid, relatively effortless, intuitive processes or (b) deliberate, slow, flexible, effortful, controlled processes [1, 7-9]; see [10] for review of dual-process as relating to prosociality.

If intuitive processes are by definition automatic, and cooperation is an acquired intuition, then we should be more prone to cooperate under contexts of time constraint (i.e., forced intuitive decisions). In contrast, when given the chance to deliberate, the extra decision-making time may override cooperative intuitions and adjust behavior toward the optimum for a given situation [1, 3]. In the context of one-shot economic encounters, in which there may be less concern about reputation or sanctions for good or bad behavior, the optimum would be to act selfishly. (By optimum, we refer to the idea that in a given situation, there is a strategy

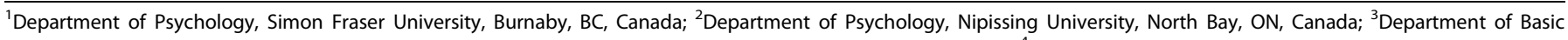

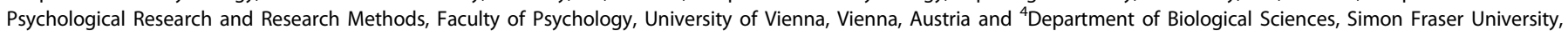
Burnaby, BC, Canada

Correspondence: Justin M. Carré (justinca@nipissingu.ca) or Neil V. Watson (nwatson@sfu.ca)

Received: 3 June 2018 Revised: 22 August 2018 Accepted: 4 September 2018

Published online: 19 September 2018 
that gives the highest expected utility. In a one-shot PGG, every dollar spent on the group project costs one dollar but yields only a private return of less than one dollar, and thus selfinterested subjects should contribute nothing to the common project [11]. Given that reputation can have a significant influence on repeated PGGs (e.g., $[12,13])$, and given that we are examining testosterone- $a$ hormone strongly implicated in status and dominance-related behaviors-we also note here that acting in a self-interested manner does not have the same potential consequences for reputation.) Some work supports this notion. For example, when participants are randomly assigned to a timeconstraint condition (i.e., make a decision in less than $10 \mathrm{~s}$ ) or a forced-delay condition (i.e., wait for at least $10 \mathrm{~s}$ before making a decision), those in the time-constraint condition make significantly greater contributions to a shared pool of resources than do those in the forced-delay condition [4]. (Some studies have failed to replicate this effect (e.g., [14-16], but see [17, 18]; see [19] for meta-analysis), which has been attributed to participant compliance (i.e., when asked to respond rapidly, some participants nevertheless deliberate, or vice versa). When restricting analyses to those who comply with instructions, as was done in the original article, the effect of time-constraint on cooperation appears robust (see [14] for a recent multi-lab replication study), with further analyses suggesting that selection biases do not properly account for the finding of significant differences in compliant-only analyses but not intent-to-treat analyses [18].)

A separate, value-based decision-making framework suggests that cognitive processes involved in cooperative behavior are not fixed, but instead hinge on individual differences [20, 21]. Specifically, it suggests that individuals with more prosocial tendencies are quicker to cooperate than they are to engage in self-interested behavior, and more self-interested individuals are quicker to engage in self-interested behavior than cooperation $[22,23]$. Indeed, some recent work supports this idea in finding that prosocial participants were intuitive cooperators, while selfish participants were deliberative cooperators [21]. Therefore, cooperative behavior may not be intuitive for every individual, and thus priming intuition or reflection may have disparate effects based on individual dispositions.

\section{EFFECTS OF TESTOSTERONE ON BEHAVIOR AND DECISION- MAKING}

Traditional wisdom suggests that testosterone is positively associated with aggressive, impulsive, and antisocial behaviors, and negatively associated with prosocial, cooperative behaviors. However, meta-analytic estimates indicate that the correlation between testosterone and human aggression is relatively weak $(r=0.08$; see [24]). Critically, more recent work suggests that testosterone's relationship to human social behavior-and decision-making more generally-may depend on social context and/or individual differences in specific personality domains. For instance, one study showed that testosterone increased the extent to which men either punished or rewarded their interaction partner, depending on whether the partner made unfair or fair offers in the ultimatum game, respectively [25]. Most relevant to intuitive decision-making, a single dose of testosterone (relative to placebo) reduced cognitive reflection among young men [26] and, in another set of studies, increased reactive aggression, but only among men high in trait dominance, low in self-control [27], or with a relatively independent self-construal [28]. Therefore, testosterone's effects on social behavior and on intuitive decision-making may depend not only on social context, but also on individual difference factors.

Combining this research on cognition and social neuroendocrinology, we aimed to address several key outstanding questions regarding cooperative behavior, including if and how testosterone influences cooperation, if and how intuition or reflection might interact with testosterone to influence cooperation, and if these (potentially synergistic) effects depend on individual differences in personality (trait dominance, self-control, self-construal). To do so, we employed a relatively large, placebo-controlled, testosterone administration study, using a sample of 400 men (betweensubjects design), and randomly assigned men to complete a oneshot public goods game (PGG) under time constraint (forced intuition) or time delay (forced reflection). Two, partially competing hypotheses guided our predictions. Based on the theoretical accounts and findings presented above, one hypothesis is that testosterone's promotion of intuitive decision-making [26] would exaggerate the prosocial effects of time-pressure [4] on cooperation $(\mathrm{H} 1)$. On the other hand, because cooperation leaves individuals vulnerable to exploitation-a potential threat to the social status/dominance [29] that testosterone is thought to promote (e.g., [30, 31]) - testosterone may reduce cooperation (as in [29]). Nevertheless, to the extent that weighing the benefits of cooperation against the status-related costs of exploitation requires cognitive effort and deliberation, we might expect these negative effects of testosterone to only exist when deliberation is possible (i.e., forced delay condition). In other words, timeconstraint may buffer against the negative effects of testosterone on cooperation $(\mathrm{H} 2)$.

Further, and regardless of whether time-constraint buffers against the negative effects of testosterone, or testosterone exaggerates the beneficial effects of time-constraint, we predicted that any interactions between intuition/deliberation and testosterone would be strongest among men high in a risk factor score comprised of previously-identified critical moderators of testosterone-behavior relationships $(\mathrm{H} 3)$, including high trait dominance, high impulsivity (low self-control), and independent self-construal (those feeling relatively disconnected from other individuals [27, 28]; see Methods section for calculation of this "Risk" score).

\section{METHODS}

Participants

The participant sample consisted of 400 healthy young men between the ages of 18 and 40 years $\left(M_{\mathrm{Age}}=22.8, \mathrm{SD}=4.7\right)$. Men were recruited in northern Ontario via online advertisements and from the online participant pool at Nipissing University, thus including students and members of the general public. Prospective participants were first screened via phone for eligibility, based on the following exclusion criteria: (1) age of less than 18 or more than 40 years, (2) participation in sports where testosterone is a banned substance, (3) taking medications known to interfere with steroid hormone concentrations, (4) drug or alcohol dependence, and/or (5) diagnosis of a mental illness. Eligible participants completed informed consent for all aspects of testing. All procedures were approved by the University's Research Ethics Board.

\section{Procedure and materials}

A procedural timeline is shown in Fig. 1. Participants were tested individually at one of the three starting times: 10:00 AM, 12:30 PM, or 2:30 PM. After providing informed consent, participants answered online demographics questions and self-report personality measures ( $25 \mathrm{~min})$. Next, a saliva sample was collected via passive drool and frozen at $-20^{\circ} \mathrm{C}$ for future assay of baseline hormone concentrations, followed by a mouthwash sample for future DNA extraction. Using a randomized, double-blind administration procedure, participants then received either $11 \mathrm{mg}$ of testosterone nasal gel (Natesto ${ }^{\mathrm{TM}}$ ), or equivalent placebo. Following drug administration, participants completed the Point Subtraction Aggression Paradigm (reported in [32]). Participants then completed the cooperation task for the current study, which was a one-shot PGG, occurring approximately $60 \mathrm{~min}$ after drug administration. 
Pre Hormone Administration

Post Hormone Administration

SAMPLES

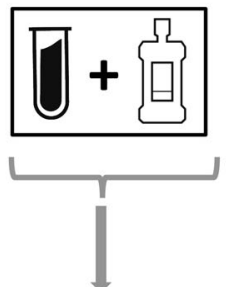

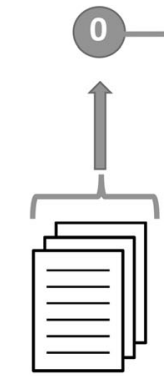

\section{CONSENT \&} SURVEYS

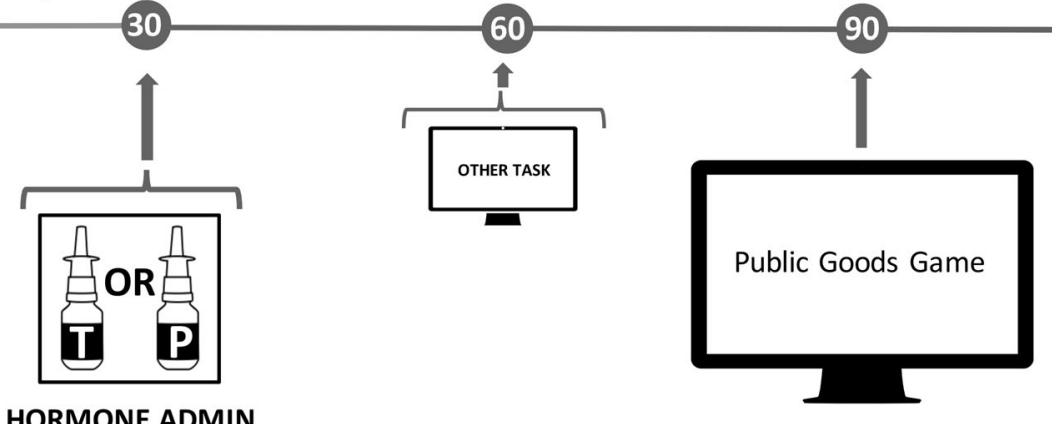

HORMONE ADMIN
SAMPLE

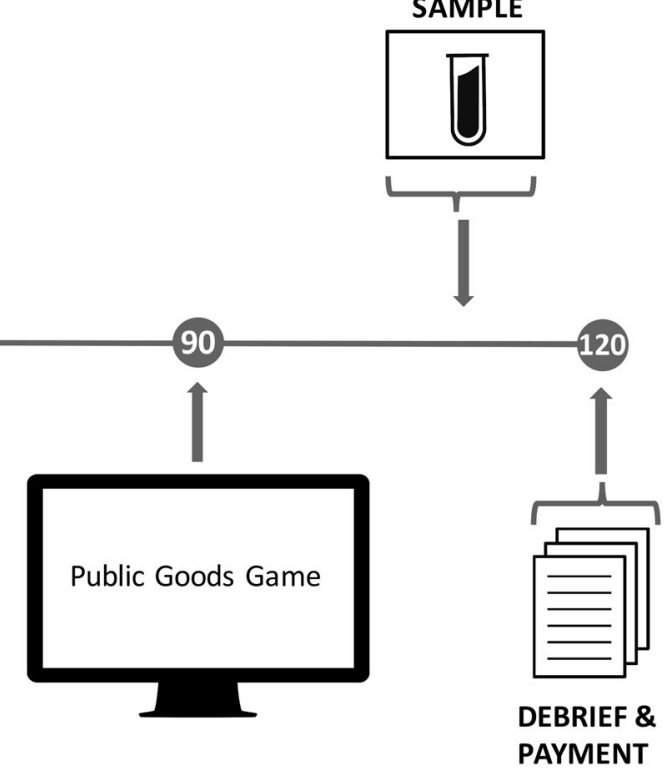

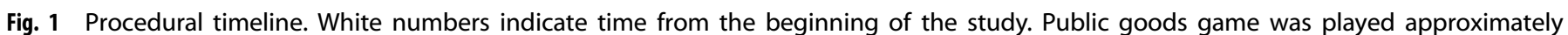

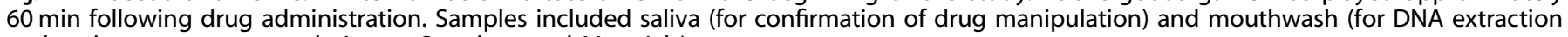
and androgen receptor analysis; see Supplemental Materials)

Personality questionnaires and the creation of a risk score Recent work in the social neuroendocrinology literature has identified three key personality moderators of the effects of testosterone on social behavior: self-control [27], self-construal $[28,33]$, and dominance [27, 34-37]. Results from these studies showed that the positive relationship between testosterone and behavior (e.g., aggression, competitive decision-making, risktaking) was strongest among individuals who were high in trait dominance, low in trait self-control (high impulsivity), or with relatively independent self-construal. Inherent in examining multiple individual moderators in a single model is a reduction in statistical power (see $[38,39]$ for review). To address this issue, the present study combined individual moderators into a more powerful "risk" score index, in line with previous works [38-40]. To achieve this, scores on each personality measure were first derived individually. Dominance scores were indexed by averaging participant responses (following standardization) on the International Personality Item Pool-Dominance [41] and the dominance subscale of the Dominance-Prestige Scale [42]; see [27] for this approach to combining these two measures. Self-control was indexed from the average of participant responses on the Barratt Impulsivity Scale (reverse-coded [43]) and the Brief Self-Control Scale [44]. Self-construal was indexed from the 24-item Self-Construal Scale [45] by subtracting the 12 "interdependent" sub-scale items from the 12 "independent" subscale items, where higher self-construal scores represent a more independent self-construal. The individual dominance, self-control (reverse-scored), and self-construal scores were then standardized and averaged to create the single risk factor score, such that higher risk score values indicated a greater risk for testosteroneinduced anti-social behavior (i.e., lower cooperation).

Hormone administration

Testosterone or placebo conditions were randomly assigned, and administration was conducted using a double-blind procedure. Men assigned to the testosterone condition received two syringes of Natesto ${ }^{\mathrm{TM}}$, each containing $5.5 \mathrm{mg}$ of gel, while men in the placebo condition received two syringes of an equivalent amount of non-active gel with similar physical properties (i.e., viscosity, color). Under the supervision of a research assistant, participants were instructed to apply the gel from one syringe to the lateral side of the left nostril, and the gel from the other syringe to the lateral side of the right nostril, and then to compress the nostrils toward the nasal septum to evenly spread the gel on nostril walls. Following administration, participants were instructed to immediately and thoroughly wash their hands in order to prevent unintentional contamination of any testing areas.

The present protocol was the first to employ the hormone methodology identified above in a sample of healthy, eugonadal men (see [46] for pharmacokinetics in hypogonadal men), and thus a pharmacokinetic pilot was first conducted in order to establish the time course in this population (see [32] for full methods). Briefly, using a separate sample of men, and a doubleblind, cross-over design (with 2-week washout), male participants $(n=13)$ had a baseline blood draw, then received $11 \mathrm{mg}$ of Natesto or equivalent placebo, followed by a blood draw at 15, 30, 60 , and $180 \mathrm{~min}$ post-administration. As expected, groups did not differ in serum testosterone concentrations at baseline, but significantly differed at all post-administration time points (all $p$ 's $<0.005$; Cohen's $d z$ range $=0.83-1.38$ ). Notably, the greatest difference in testosterone concentrations occurred at $60 \mathrm{~min}$ post administration - the time at which behavioral testing for the current study was conducted.

Time pressure versus time delay public goods game The PGG is a widely-used social dilemma paradigm for measuring cooperation among group members. Cooperation, as defined in the scientific literature and as used for the present study, is considered an act where one individual pays a cost for another to receive a benefit [47]. The PGG has been used for decades as a standard paradigm for measuring cooperation, as the nature of the game is an operationally-defined cooperation task: individuals in the PGG have the opportunity to pay a cost for the benefit of other group members. In a traditional PGG, participants are given 
a starting endowment and then tasked with deciding how much to give to a "common project", and how much to keep for themselves. Participants are told that all amounts given to the common project are multiplied by a factor (often tripled) and then split evenly among the members. Participant decisions are made in the context of contrasting optimal outcomes for themselves versus optimal outcomes for the group. In other words, the optimal strategy for an individual is not necessarily the optimal strategy for the group, and thus participants must decide whether to cooperate/act prosocially (i.e., give more money to the common project) or to not cooperate/act more selfishly (i.e., give less money to the common project [48]).

For the present study, we employed a version of the PGG used in previous work to study the effects of time-pressure or timedelay on cooperation $[4,14]$, which was delivered using the online Qualtrics program (Qualtrics, Provo, UT), with all instructions presented on screen. Participants were first told that they would be playing a decision-making game with three other randomly assigned members of the study (in actuality, the computer program). It was then explained that the amount of money they earned from the game would depend on their own decisions and the decisions of the other members of the group. Prior to playing, each participant was given a \$4 (400 cents) endowment and was provided with examples of potential outcomes of the game.

Participants were then randomly assigned to the time-pressure condition or the forced-delay condition. Instructions were identical to those from [14], with added bold capitalization to ensure saliency. In the time-pressure condition, participants' screens read "You must MAKE YOUR DECISION IN LESS THAN 10 SECONDS!" In the forced-delay condition, participants' screens read "Please carefully consider your decision. You must WAIT AND THINK FOR AT LEAST 10 SECONDS BEFORE MAKING YOUR DECISION!" Consistent with [14], a timer was shown on screen so that participants were aware of (a) the time left to make a decision in the time-pressure condition (i.e., a timer counting down from 10), or (b) the time left to wait before making a decision in the forced-delay condition (i.e., a timer counting up from 10). Participants selected their contribution amounts using an on-screen slider ranging from 0 cents to 400 cents, with the slider starting in the middle position (no value was marked for the middle position). The program recorded each participant's contribution amount and the time at which they submitted their contribution. After participants made their decision, the results of their game were presented, including their own actual contribution amount, and the fictitious contributions of the ostensible other three group members.

Saliva collection and pharmacokinetic manipulation check In addition to the pharmacokinetic study described above, we further verified that the administration procedure boosted participants' testosterone concentrations via collection of saliva samples at baseline (pre-hormone administration), and at the end of the study ( 80 min post-administration). Saliva was collected via passive drool into a $5 \mathrm{ml}$ polystyrene tube, and was subsequently frozen at $-20^{\circ} \mathrm{C}$. Samples were later thawed and centrifuged, followed by extraction of the supernatant. Samples were assayed in duplicate using commercially available enzyme immunoassay kits from DRG International (coefficients of variation: intra-assay = $8.45 \%$; inter-assay $=12.46 \%$ ).

\section{Analytic approach}

With censored data, as commonly found in PGG investigations, ordinary least squares regression approaches can be unduly influenced by a greater number of scores in the tail(s) of the distribution. To address this potential issue, and consistent with prior work examining the effects of time-pressure and delay in a one-shot PGG paradigm [4], main analyses of interest were conducted using a Tobit regression approach with robust standard errors, which allows for estimation of scores beyond the maximum response option available (i.e., 400 cents) while simultaneously accounting for potential heteroskedasticity in the residuals. Tobit regressions were conducted using the survival package for $\mathrm{R}$ (version 2.38; [49]). We note, however, that using a simple linear regression approach yielded similar results.

The risk score variable was first standardized, allowing the unstandardized regression coefficients to represent the extent of change in cooperation (PGG contribution) for a one standard deviation increase in risk factor score. [For additional analyses presented with individual moderators (i.e., self-control, selfconstrual, and dominance), the same approach was used, where the unstandardized regression coefficient represented the change in PGG contributions for one standard deviation increase in the moderator. Individual moderator analyses are presented in the Supplemental Materials]. The drug variable was similarly centered at zero, but with a one-unit distance between the testosterone and placebo conditions; thus, the unstandardized regression coefficients for drug represent the difference in PGG contributions between those who received testosterone versus those who received placebo. Follow-up conditional effects or simple slope analyses, where indicated, were conducted at relatively low ( $-1 \mathrm{SD})$ and relatively high (+1 SD) levels of risk factor. In line with a recent multi-lab replication attempt [14] of Rand et al.'s original work [4], we present both intent-to-treat analyses, which involve all participants, regardless of whether they followed the time condition instructions, as well as compliant-only analyses, which involve only participants who correctly followed the instructions for their respective time condition (i.e., if in the time-pressure condition, made their contribution in less than $10 \mathrm{~s}$; if in the forced-delay condition, waited at least $10 \mathrm{~s}$ before making their contribution).

\section{RESULTS}

Testosterone manipulation check

Analysis of salivary testosterone confirmed that the manipulation was effective $\left(t_{397}=6.17, p<0.001\right.$, Cohen's $d=0.62$ for the difference in concentrations between placebo and testosterone groups following administration, measured at the end of the study), and as expected, the groups did not differ prior to administration ( $t_{395}=0.25, p=0.80$, Cohen's $d=0.03$ ). Further, participants were no better than chance $(p=0.32)$ at guessing the drug condition to which they were assigned (testosterone or placebo; guess correct $=47.4 \%$ of sample), suggesting they were not consciously aware of which substance they had received.

Effects of drug, time condition, and risk factor

Intent-to-treat analyses. An intent-to-treat analysis examining the effects of drug (testosterone or placebo), time condition (timepressure or forced delay), risk factor (individual difference variable), and their interactions revealed no main effects of drug $(b=-18.91, p=0.48)$, or risk factor $(b=-33.17, p=0.22)$. The time condition effect was in the predicted direction of the social heuristic hypothesis [4], but not statistically significant (timepressure contributions $>$ forced-delay contributions, $b=-33.17$, $p=0.22)$. No significant two-way interactions were identified for drug $\times$ time condition $(b=-6.33, p=0.91)$, drug $\times$ risk factor $(b=-18.18, p=0.49)$, or time condition $\times$ risk factor $(b=24.57$, $p=0.35)$. Notably, there was a significant drug $\times$ time condition $\times$ risk factor interaction $(b=-191.88, p<0.001$, see Fig. 2). Followup analyses revealed that the drug $\times$ time condition interaction was significant for men with relatively high risk factor scores $(b=$ $-198.20, p=0.007)$, and for men with relatively low risk factor scores $(b=185.549, p=0.017)$. Among men high in risk, testosterone decreased PGG contributions in the forced delay condition $(b=-136.19, p=0.007)$, but did not affect contributions in the time-pressure condition $(b=62.01, p=0.24)$. Among 
Low Risk Men

700

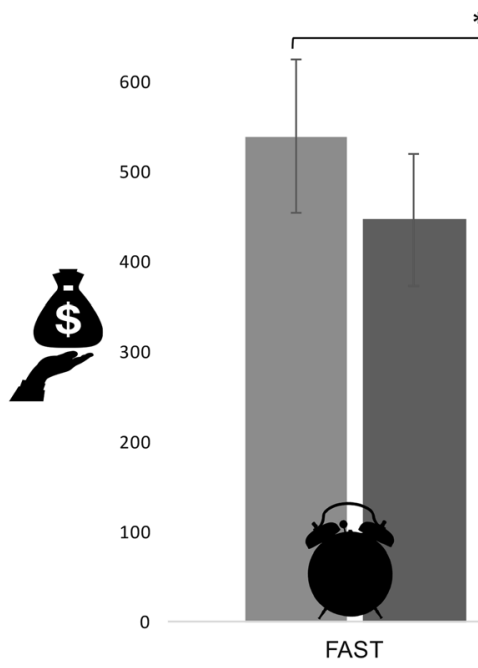

Placebo

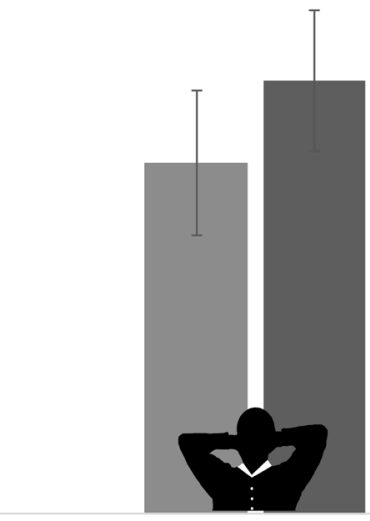

SLOW
High Risk Men

Testosterone

Fig. 2 Effect of drug (testosterone versus placebo) on contributions in the public goods game (PGG) as a function of time condition (forced intuition $=$ FAST; forced reflection $=$ SLOW) and risk factor score (total $N=400$; placebo $=201$ [fast $=100$, slow $=101]$, testosterone $=199$ $[$ fast $=99$, slow $=100]$ ). The $Y$-axis represents the Tobit model predicted PGG contribution values (note that Tobit estimates a latent variable beyond the maximum 400 cents, allowing a more reliable estimate). Error bars represent the $95 \%$ confidence intervals. For men high in risk $(+1 \mathrm{SD})$, testosterone significantly decreased contributions in the forced reflection (SLOW) condition $(p=0.007)$ but not the time-pressure (FAST) condition $(p=0.24)$. For men low in risk $(-1 \mathrm{SD})$, the effect of testosterone did not differ between the time-pressure condition and the forced reflection condition. Additional contrasts showed that high risk men receiving testosterone contributed significantly less in the forced reflection condition than the time-pressure condition $(p=0.015)$, whereas high risk men receiving placebo contributed significantly less in the time-pressure condition than the forced reflection condition $(p=0.016)$. For low risk men receiving placebo, contributions were significantly higher in the time-pressure condition than the forced reflection condition $(b=-243.286, p=0.007)$, but this difference was abolished by testosterone $(b=127.81, p=0.13)$

men low in risk, however, there were no significant drug effects in either the forced delay $(b=92.04, p=0.09)$ or the time-pressure condition [47] $(b=-93.51, p=0.09)$. [Conducting the same analyses while controlling for participants' performance on the Point Subtraction Aggression Paradigm [32] performed earlier in the protocol left the findings unchanged, as did controlling for whether participants believed they had received testosterone or placebo, or for session testing time (see Supplementary Materials).] Additional post-hoc contrasts revealed that among men high in risk who received placebo, contributions were significantly lower in the time-pressure condition than the forced delay condition $(b=189.60, p=0.016)$, whereas among men high in risk who received testosterone, contributions were significantly lower in the forced delay condition than in the time-pressure condition $(b=-206.81, p=0.015)$. Among low risk men receiving placebo, contributions were significantly higher in the timepressure condition than the forced delay condition $(b=-243.286$, $p=0.007)$, but among low risk men receiving testosterone, this effect was abolished $(b=127.81, p=0.13)$.

Compliant-only analyses. When we conducted the same analyses above but restricted our sample to men who followed the timing instructions ( $n=282,70.5 \%$ of the full sample) we found no main effect of Drug $(b=-40.98, p=0.21)$ or risk factor $(b=-16.59, p$ $=0.29$ ), but a significant main effect of time condition (timepressure contributions $>$ forced delay contributions; $b=-172.41$, $p<0.001)$. No significant two-way interaction effects were noted (| $\left.b^{\prime} s \mid=7.01-50.51, p^{\prime} s=0.19-0.82\right)$. Consistent with the intent-totreat analysis, the drug $\times$ time condition $\times$ risk factor interaction was significant $(b=-213.08, p<0.001)$. Follow-up analyses again showed that among men with relatively high risk factor scores, the drug $\times$ time condition interaction was significant $(b=-263.59, p$ $=0.002$ ), such that testosterone decreased contributions in the forced-delay condition $(b=-165.76, p=0.003)$ but did not differ from placebo in the time-pressure condition $(b=97.83, p=0.14)$. Among men with relatively low risk factor scores, the drug $\times$ time condition interaction did not reach statistical significance $(b=162.57, p=0.09)$. [Given arguments that differences in the effect of cooperation between intent-to-treat analyses and compliant-only analyses may be due to selection biases (e.g., compliant individuals may have more of a particular trait [14]), we tested whether there were differences in individual dispositional qualities between those who followed instructions (compliant) and those who did not (non-compliant). No differences were found between the groups for self-construal $(t(398)=-0.72$, $p=0.47)$, dominance $(t \quad(398)=-0.35, p=0.72)$, self-control $(t(398)=0.34, p=0.73)$, or risk factor score $(t(398)=-0.73$, $p=0.47)$. See Supplemental Materials for descriptive statistics and bivariate correlations]. Additional post-hoc contrasts revealed that among men high in risk who received placebo, there was no difference in contributions between the time-pressure condition and the forced delay condition $(b=119.17, p=0.19)$, whereas among high risk men receiving testosterone, contributions were significantly lower in the forced delay condition than in the time-pressure condition $(b=-385.99, p<0.001)$. Among low risk men receiving placebo, contributions were significantly higher in the time-pressure condition than the forced delay condition $(b=-371.72, p<0.001)$, but-as with the intent-totreat analysis-among low risk men receiving testosterone, this effect was abolished $(b=-53.88, p=0.59)$.

\section{DISCUSSION}

Using placebo-controlled administration of exogenous testosterone in a relatively large sample of healthy young men, we have examined several novel aspects of the expression of cooperative behaviors, and specifically: if and how men's cooperation is influenced by exogenous testosterone in a time-pressure or time- 
delayed one-shot PGG, and if the effects of testosterone on men's cooperation in this context vary with individual differences in specific dispositional qualities (dominance, self-control, and selfconstrual). Findings revealed that (1) within the placebo group, time-pressure increased cooperation among low risk men, but decreased cooperation among high risk men; (2) testosterone moderated this pattern by abolishing the time-pressure effect among low risk men, and-in high risk men-reversing the effect by selectively reducing offers under forced delay. The cooperation-reducing effects of testosterone after a forced-delay were robust to whether we analyzed all participants, or restricted analyses to only those who complied.

It has been argued that deliberation serves to override cooperative intuitions, thus facilitating the adjustment of behavior toward the optimum for a given situation-which, in a one-shot encounter, would be to act selfishly $[1,3]$. The results here do not support the idea that time-pressure alone can induce cooperative efforts, but are instead consistent with the idea that individual differences in disposition are important, and that testosterone can play a role in promoting behavior that is more immediately advantageous to the individual, particularly for men with high dominance, low self-control, and independent self-construals. That the effects of testosterone on cooperation depended on these personality variables contributes to an emerging literature suggesting that the effects of testosterone in various socialbehavioral domains; (e.g., aggression, competitive decision making, risk-taking $[27,28,32-34])$ vary as a function of one's dispositional qualities.

Importantly, our results are consistent with a value-based decision-making framework suggesting that prosocial behavior may not be intuitive for everyone. Wills et al. [21] found that prosocial participants were intuitive cooperators, whereas more selfish participants were deliberative cooperators. Our findings reflect this idea: among men low in risk for antisocial behavior, priming intuition was associated with more prosocial contributions than deliberation; among men high in risk for antisocial behavior, however, priming deliberation was associated with higher contributions than intuition. Thus, our results suggest that individuals with low risk profiles may be intuitive cooperators, whereas individuals with high risk profiles may be deliberative cooperators, and that testosterone shifts these relationships such that intuitive cooperation among low risk men is abolished by testosterone, and deliberative cooperation among high risk men is reversed to deliberative defection.

Testosterone is argued to facilitate life history trade-offs, including survival versus reproduction, and mating versus parenting effort $[50,51]$. In line with this idea, testosterone fluctuates rapidly in response to evolutionarily-salient stimuli, such as competitive wins or losses $[52,53]$, and interactions with potential mates [54]; these fluctuations, in turn, map onto future behavior in the same life history domains (see [55] for review). Transcending these domains is testosterone's role in promoting the striving for, and maintenance of, status. It is possible that status concerns involve thoughts about the potential outcomes of the interaction, such as whether or not someone will exploit the contributor(s) [29]. Although speculative, testosterone may have reduced contributions in high-risk men as a means of protection against such exploitation and its potential negative consequences on status/dominance. Notably, this status-protection strategy may only be favored in the context of a one-shot, anonymous PGG, as was used here. In situations with repeated interactions with the same individual(s), or where the decisions are not anonymous (and reputational information can spread), there would be additional incentive to maintain a good reputation and contribute, despite concerns about exploitation. Indeed, individuals known by the group to contribute more (versus less) are more respected and ascribed higher status within the group (e.g., [56]), and including a reputational component to repeated social dilemma games encourages cooperation $[12,13]$. Therefore, when reputational information can spread, the beneficial effects of contributing may outweigh the negative effects of being exploited. This remains one hypothesis, which will require a direct test in future work. Other possibilities exist, and studies may want to compare them against the idea of reputational concerns. For example, it is possible that in the forced-delay condition, and without any prior information of the other players' contribution styles, high risk men on testosterone may have become less trustworthy of the other players, thus influencing contributions. [Trust may, however, be part of a more complex pathway to reduced contributions, where testosterone leads to reduced trust, leading to exploitation concerns, and thus reduced contributions.] Some work has found that changes in men's testosterone can reduce trust in emotionally neutral faces [57]; but see [58], and among women, can reduce trust in game partners [29], suggesting this possibility here. The exact mechanisms by which testosterone, personality, and reflection interact to predict changes in cooperative behavior in anonymous encounters will require future work, and may be strengthened by a contrast with a repeated trial PGG.

Testosterone's effects may also change depending on whether someone is directly watching the encounter, and particularly someone relevant to mating (e.g., an attractive member of the opposite sex). Evidence indicates that men's cooperation can indeed be modulated by observer status: contributions are higher in the presence of an attractive female, and also correspond to the degree to which men find the female observer attractive, arguably because of reputational status concerns that may influence mating opportunities [59]. If testosterone promotes mating effort and status-seeking behaviors, the presence of a potential mate might dictate that a rise in testosterone from exogenous administration would exaggerate effects normally seen from observer status alone. Further, such effects might be particularly strong among men who have dispositions oriented towards status in the first place (e.g., highly dominant). Future research will be important for answering this question.

Given recent debates about the existence of intuitive cooperation effects $[3,4,14-19]$, the present study allowed a further test of the social heuristic hypothesis. With an intent-to-treat approach, there was no main effect of time condition, although the effect was in the predicted direction (time-pressure contributions $>$ forced-delay contributions). However, when restricting analyses to those who followed the instructions, contributions were significantly higher under time pressure than delay. Notably, the presence of a time-pressure effect for compliant-only individuals is consistent both with Rand et al.'s original work [4] and a recent multi-lab replication [14]. Rand et al. [4] found that when examining individuals who followed the instructions, timepressure predicted increased cooperation relative to forced delay. Bouwmeester et al. [14] also found this same effect, but showed that the effect disappeared in the intent-to-treat analysis. Bouwmeester et al. had relatively low rates of compliance with the instructions for the time-pressure condition (34.1\%), which Rand [18] notes leaves the possibility that a causal effect of timepressure on cooperation still exists. The present study had considerably higher rates of compliance in the time-pressure condition (80.9\%), but still failed to find a time-pressure effect in the intent-to-treat analysis. It has been argued that intent-to-treat analyses may introduce biases by selecting for individuals who are cooperative in the first place [14], but here we found no differences in personality between compliant and noncompliant individuals (see also [18]). Further, our interactive effects of testosterone and dispositional risk in the forced delay condition were robust to whether participants were compliant with timing instructions, allowing greater confidence in the effect. Nevertheless, we also provide evidence to support a value-based framework, such that cooperative strategies differ based on individual differences in personality risk, and thus suggests that 
previous inconsistencies in support for the social heuristic hypothesis may be a function of failing to consider specific dispositional characteristics of participants.

\section{LIMITATIONS AND FUTURE DIRECTIONS}

The present study focused exclusively on men. While this sexspecific focus may be considered a strength among a greater number of testosterone administration studies examining women, the question remains as to how the effects presented here might generalize to women. To date, the testosterone literature for women shows results that might be considered contradictory (e.g., increased cooperation following testosterone administration [60]; although see [29]), but it is not clear whether differences for studies with women might be influenced by the supraphysiological levels of testosterone that are typically reached in the samples, differences in one shot paradigms versus those involving multiple encounters with the same individual, differences in participant beliefs about the effects of testosterone (e.g., [60]), or some other variable. Future research examining men and women in the same study, where allowed by regulatory jurisdictions, would be beneficial for disentangling any potential sex differences.

Mental health screening was conducted via phone in the initial phone interview process, and as per our exclusionary criteria, any individual who self-reported a developmental disability or psychological disorder was not eligible for the study. A limitation to this approach is that we could not independently verify diagnoses (e.g., via formal clinical diagnostic interview, review of medical records). Future studies could include more formal assessment of mental health disorders to increase the likelihood of accurate detection.

The majority of previous works have conducted behavioral testing approximately $4 \mathrm{~h}$ post administration in women $[55,61]$ following a study showing that the effects of sublingual testosterone administration on women's vaginal pulse amplitude in response to sexual stimuli emerged at $4 \mathrm{~h}$ post administration [62]. However, previous work from our lab examining men has found that a single administration of testosterone can increase threat-related amygdala, hypothalamic, and periaqueductal gray reactivity to angry facial expressions within 90 min post administration [63], and effects of testosterone on aggressive behavior are found within $60 \mathrm{~min}$ post administration [27]. We also find similar behavioral effects from exogenous testosterone administration on men's preferences for feminine faces when tested at 2 and $3 \mathrm{~h}$ post administration [64], as well as effects on selfperceived dominance tested at 2 and $4 \mathrm{~h}$ post administration [65]. That effects in the present study are found within 60 min suggests the possibility of a non-genomic mechanism, similar to findings in animal models with rapid effects of testosterone on brain function and behavior (reviewed in [66]). The possibility exists that the effects of testosterone and personality on cooperation would be found at even earlier time points (e.g., 15-30 min post administration), as we found a significant increase in testosterone within 15 min of administration. Future work may want to examine this possibility.

Our hypotheses were partially informed by the idea that testosterone can promote impulsive behavior, such as reactive aggression. While testosterone did seem to increase cooperation in the time-pressure condition for high risk men, it did not reach statistical significance. It is possible that cooperation as an impulsive/intuitive behavior is different than impulsive aggression following provocation. Given that testosterone is highly implicated in social dominance behaviors, and reflexive dominance behaviors are often triggered by direct social threats (reviewed in [67]), testosterone may simply not function in the same manner in the context of having to make a cooperative decision under timepressure as it does when an individual is directly provoked. A further distinction may be made between the one-shot PGG and paradigms typically used to examine aggressive responses. In the one-shot PGG, participants interact with "other participants" on one occasion only, and thus there is no risk for retaliation and/or social sanctions. In aggression paradigms like the PSAP, however, interactions with other participants are longer and dynamic, with many opportunities for retaliation throughout the task. Dreher et al. [25] found that testosterone increased both prosocial and antisocial status-enhancing behaviors in human men, but notably, their experiment involved repeated ultimatum games, where testosterone increased prosocial behavior only following the receipt of large offers from their game partner. Therefore, in a oneshot PGG, testosterone may not predict impulsive prosociality, but may in a context of available information about game partners. It may be useful for future studies to employ exogenous testosterone administration in the context of a repeated PGG with timepressure and forced-delay manipulations to allow a test of this hypothesis.

\section{ACKNOWLEDGEMENTS}

We would like to thank Amy Mattsson, Ben Zao, Brandon Reimer, Brittney Robinson, Caelan Mathers, Camille Gray, Chad Tremblay, Charlotte Miller, Christine Jessamine, Dalton Rogers, Emily Jeanneault, James Donovan, Jessica Cearns, Jessica Ibey, Jordan McVittie, Kendra Maracle, Kim Gilbert, Kirsten Loates, Megan Phillips, Nadia Desrosiers, and Victoria Bass-Parcher for data collection. We thank Dr. Bernie Crespi for the use of his laboratory and reagents for genetic assay. We also thank Emily Ceretti, a registered pharmacist, and her team at Health Care Pharmacy for preparing the placebo and Natesto syringes. This project was funded by a Natural Sciences and Engineering Research Council of Canada (NSERC) Discovery Grant (RGPIN-201406676) and Northern Ontario Heritage Fund Corporation to JMC, and an NSERC Discovery Grant (RGPIN-2016-05706) to NVW. BMB holds a Joseph-Armand Bombardier Doctoral Scholarship from the Social Sciences and Humanities Research Council (SSHRC) of Canada. Funding sources played no role in the conceptualization, design, data collection, analysis, decision to publish, or preparation of the manuscript.

\section{ADDITIONAL INFORMATION}

Supplementary Information accompanies this paper at (https://doi.org/10.1038/ s41386-018-0220-8).

Competing interests: The authors declare no competing interests.

Publisher's note: Springer Nature remains neutral with regard to jurisdictional claims in published maps and institutional affiliations.

\section{REFERENCES}

1. Bear A, Rand DG. Intuition, deliberation, and the evolution of cooperation. Proc Natl Acad Sci USA. 2016;113:936-41.

2. Cone J, Rand DG. Time pressure increases cooperation in competitively framed social dilemmas. PLoS ONE. 2014;9:1-13.

3. Rand DG, Peysakhovich A, Kraft-Todd GT, Newman G, Wurzbacher O, Nowak MA, et al. Social heuristics shape intuitive cooperation. Nat Commun. 2014;5:1-12.

4. Rand DG, Greene JD, Nowak MA. Spontaneous giving and calculated greed. Nature. 2012;489:427-30.

5. Andreoni J. Impure altruism and donations to public goods: a theory of warmglow giving. Econ J. 1990;100:464-77.

6. Crumpler H, Grossman PJ. An experimental test of warm glow giving. J Public Econ. 2008;92:1011-21.

7. Kahneman D. A perspective on judgment and choice. Am Psychol. 2003;58:697720.

8. Sloman SA. The empirical case for two systems of reasoning. Psychol Bull. 1996;119:3-22

9. Stanovich KE, West RF. Individual differences in rational thought. J Exp Psychol Gen. 1998:2:161-88.

10. Zaki J, Mitchell JP. Intuitive prosociality. Curr Dir Psychol Sci. 2013;22:466-70.

11. Camerer CF, Fehr E. Measuring social norms and preferences using experimental games: a guide for social scientists. Found Hum Soc. 2004;97:55-95.

12. Mcintosh C, Sadoulet E, Buck S, Rosada T. Reputation in a public goods game: taking the design of credit bureaus to the lab. J Econ Behav Organ. 2013;95:270-85. 
13. Pfeiffer T, Tran L, Krumme C, Rand DG. The value of reputation. J R Soc Interface. 2012;9:2791-7.

14. Bouwmeester S, Verkoeijen PP, Aczel B, Barbosa F, Bègue L, Brañas-Garza P, et al. Registered Replication Report: Rand, Greene, and Nowak (2012). Perspect Psychol Sci. 2017;12:527-42.

15. Verkoeijen PPJL, Bouwmeester S. Does intuition cause cooperation? PLoS ONE. 2014;9:e96654.

16. Tinghög G, et al. Intuition and cooperation reconsidered. Nature. 2013;498:5-8.

17. Rand DG, Green JD, Nowak MA. Rand et al. reply. Nature. 2013;498:E2.

18. Rand DG. Reflections on the time-pressure cooperation registered replication report. Perspect Psychol Sci. 2017;12:543-7.

19. Rand DG. Cooperation, fast and slow: meta-analytic evidence for a theory of social heuristics and self-interested deliberation. Psychol Sci. 2016;27:1192-206.

20. Levy DJ, Glimcher PW. The root of all value: a neural common currency for choice. Curr Opin Neurobiol. 2012;22:1027-38.

21. Wills J, Hackel L, Van Bavel JJ. Shifting prosocial intuitions: neurocognitive evidence for a value-based account of group-based cooperation; 2018.

22. Krajbich I, Bartling B, Hare T, Fehr E. Rethinking fast and slow based on a critique of reaction-time reverse inference. Nat Commun. 2015;6:7455.

23. Hutcherson CA, Bushong B, Rangel AA. Neurocomputational model of choice and its implications. Neuron. 2015;87:451-62.

24. Archer J, Graham-Kevan N, Davies M. Testosterone and aggression: a reanalysis of Book, Starzyk, and Quinsey's (2001) study. Aggress Violent Behav. 2005;10: 241-61.

25. Dreher J-C, Dunne S, Pazderska A, Frodl T, Nolan JJ, O'Doherty JP. Testosterone causes both prosocial and antisocial status-enhancing behaviors in human males. Proc Natl Acad Sci USA. 2016;113:11633-8.

26. Nave G, Nadler A, Zava D, Camerer C. Single dose testosterone administration impairs cognitive reflection in men. Psychol Sci. 2017;28:1398-407.

27. Carré JM, Geniole SN, Ortiz TL, Bird BM, Videto A, Bonin PL. Exogenous testosterone rapidly increases aggressive behavior in dominant and impulsive men Biol Psychiatry. 2017;82:249-56.

28. Welker KM, Norman RE, Goetz S, Moreau BJ, Kitayama S, Carré JM. Preliminary evidence that testosterone's association with aggression depends on selfconstrual. Horm Behav. 2017:92:117-27.

29. Boksem MA, Mehta PH, Van den Bergh B, van Son V, Trautmann ST, Roelofs $K_{,}$ et al. Testosterone inhibits trust but promotes reciprocity. Psychol Sci. 2013;24: 2306-14.

30. Eisenegger C, Haushofer J, Fehr E. The role of testosterone in social interaction. Trends Cogn Sci. 2011;15:263-71.

31. Mazur A, Booth A. Testosterone and social dominance in men. Behav Brain Sci. 1998;21:353-97.

32. Geniole SN, Procyshyn T, Marley N, Ortiz TL, Bird BM, Marcellus AL, et al. Using a psychopharmacogenetic approach to identify the pathways through which and people for whom testosterone promotes aggression. Psychol Sci.2018; in press.

33. Welker KM, Roy ARK, Geniole S, Kitayama S, Carré JM. Taking risks for persona gain: an investigation of self-construal and testosterone responses to competition. Soc Neurosci. 2017;0:1-15.

34. Pranjal A, Mehta H, Van Son V, Welker KM, Prasad S. Exogenous testosterone enhances and inhibits competitive decision-making depending on victorydefeat experience and trait dominance. Psychoneuroendocrinology. 2015;60: $1-24$.

35. Carré JM, Putnam SK, McCormick CM. Testosterone responses to competition predict future aggressive behaviour at a cost to reward in men. Psychoneuroendocrinology. 2009;34:561-70.

36. Slatcher RB, Mehta PH, Josephs RA. Testosterone and self-reported dominance interact to influence human mating behavior. Soc Psychol Pers Sci. 2011;2:531-9.

37. Knight $\mathrm{EL}$, et al. Exogenous testosterone enhances cortisol and affective responses to social-evaluative stress in dominant men. Psychoneuroendocrinology. 2017;85:151-7.

38. Kraemer HC. Discovering, comparing, and combining moderators of treatment on outcome after randomized clinical trials: a parametric approach. Stat Med. 2013;32:1964-73.

39. Wallace ML, Frank E, Kraemer HC. A novel approach for developing and interpreting treatment moderator profiles in randomized clinical trials. JAMA Psychiatry. 2013;70:1241-7.

40. Evans GW, Li D, Whipple SS. Cumulative risk and child development. Psychol Bull. 2013;139:1342-96.
41. Goldberg LR, Johnson JA, Eber HW, Hogan R, Ashton MC, Cloninger CR, et al The international personality item pool and the future of public-domain personality measures. J Res Pers. 2006;40:84-96.

42. Cheng JT, Tracy JL, Foulsham T, Kingstone A, Henrich J. Two ways to the top: evidence that dominance and prestige are distinct yet viable avenues to social rank and influence. J Pers Soc Psychol. 2013;104:103-25.

43. Patton JH, Stanford MS, Barratt ES. Factor structure of the Barratt impusliveness scale. J Clin Psychol. 1995;51:768-74.

44. Tangney JP, Baumeister RF, Boone AL. High self-control predicts good adjustment, less pathology, better grades, and interpersonal success. J Pers. 2004;72: 271-324.

45. Singelis TM. The measurement of independent and interdependent selfconstruals. Pers Soc Psychol Bull. 1994;20:580-91.

46. Rogol A, Tkachenko N, Bryson N. Natesto ${ }^{\mathrm{TM}}$, a novel testosterone nasal gel, normalizes androgen levels in hypogonadal men. Andrology. 2016;4:46-54.

47. Rand DG, Nowak MA. Human cooperation. Trends Cogn Sci. 2013;17:413-25.

48. Archetti M, Scheuring I. Review: game theory of public goods in one-shot social dilemmas without assortment. J Theor Biol. 2012;299:9-20.

49. R Core Team. R: a language environment for statistical computing; 2016.

50. Del Giudice M, Gangestad SW, Kaplan HS. Life history theory and evolutionary psychology. In: Handbook of evolutionary psychology; 2015. p. 88-114.

51. Hau M, Wingfield JC Hormonally-regulated trade-offs: evolutionary variability and phenotypic plasticity in testosterone signaling pathways. In: Mechanism of life history evolution; 2008. p. 349-61.

52. Carré JM, Olmstead NA. Social neuroendocrinology of human aggression: examining the role of competition-induced testosterone dynamics. Neuroscience. 2015;286:171-86.

53. Geniole SN, Bird BM, Ruddick EL, Carré JM. Effects of competition outcome on testosterone concentrations in humans: an updated meta-analysis. Horm Behav. 2017;92:37-50.

54. Roney JR, Lukaszewski AW, Simmons ZL. Rapid endocrine responses of young men to social interactions with young women. Horm Behav. 2007;52:326-33.

55. Zilioli S, Bird BM. Functional significance of men's testosterone reactivity to social stimuli. Front Neuroendocrinol. 2017;47:1-18.

56. Hardy CL, Van Vugt M. Nice guys finish first: the competitive altruism hypothesis Pers Soc Psychol Bull. 2006;32:1402-13.

57. Carré JM, Baird-Rowe CD, Hariri AR. Testosterone responses to competition predict decreased trust ratings of emotionally neutral faces. Psychoneuroendocrinology. 2014;49:79-83.

58. Bird BM, Geniole SN, Little AC, Moreau BJ, Ortiz TL, Goldfarb B, et al. Does exogenous testosterone modulate men's ratings of facial dominance or trustworthiness? Adapt Hum Behav Physiol. 2017;3:365-85.

59. Van Vugt $M$, Iredale W. Men behaving nicely: public goods as peacock tails. Br J Psychol. 2013;104:3-13.

60. Eisenegger $C$, Naef M, Snozzi R, Heinrichs M, Fehr E. Prejudice and truth about the effect of testosterone on human bargaining behaviour. Nature. 2010;463: 356-9.

61. Bos PA, Panksepp J, van Honk J. Acute effects of steroid hormones and neuropeptides on human social-emotional behavior: a review of single administration studies. Front Neuroendocrinol. 2012;33:17-35.

62. Tuiten A, van Honk J, Koppeschaar H, Bernaards C, Thijssen J, Verbaten R. Time course effects of testosterone administration on sexual arousal in women. Arch Gen Psychiatry. 2000;57:149-53.

63. Goetz SM, Tang L, Thomason ME, Diamond MP, Hariri AR, Carré JM. Testosterone rapidly increases neural reactivity to threat in healthy men: a novel two-step pharmacological challenge paradigm. Biol Psychiatry. 2014;76: 324-31.

64. Bird BM, Welling LLM, Ortiz TL, Moreau BJ, Hansen S, Emond M, et al. Effects of exogenous testosterone and mating context on men's preferences for female facial femininity. Horm Behav. 2016;85:76-85.

65. Welling LLM, Moreau BJ, Bird BM, Hansen S, Carré JM. Exogenous testosterone increases men's perceptions of their own physical dominance. Psychoneuroendocrinology. 2016;64:136-42.

66. Foradori CD, Weiser MJ, Handa RJ. Non-genomic actions of androgens. Front Neuroendocrinol. 2008;29:169-81.

67. Terburg D, van Honk J. Approach-avoidance versus dominance-submissiveness: a multilevel neural framework on how testosterone promotes social status. Emot Rev. 2013;5:296-302. 